\title{
Repeat renal biopsy in lupus nephritis - unnecessary harm and risk of complications or important diagnostic tool with clinical consequences?
}

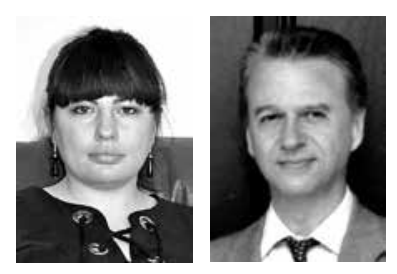

\author{
Ewa Haładyj ${ }^{1}$, Ricard Cervera ${ }^{2}$ \\ ${ }^{1}$ Department of Connective Tissue Diseases, National Institute of Geriatrics, Rheumatology and Rehabilitation, Warsaw, Poland \\ 2Department of Autoimmune Diseases, Hospital Clinic, Barcelona, Catalonia, Spain
}

Despite many years of experience and numerous studies, lupus nephritis (LN) management still remains a challenge for clinicians. The choice of diagnostic procedures and subsequent optimal therapy in the population of patients with LN is crucial to achieve remission. Renal biopsy has an important role in the diagnostic process at the beginning of the disease, and its performance at diagnosis of $L N$ is not a subject of controversy. However, the performance of biopsy in a subsequent renal flare or after maintenance treatment is not common.

According to the current standard of care in LN, after the induction treatment, maintenance therapy with mycophenolate mofetil (MMF) or azathioprine (AZA) for at least 3 years is indicated. However, a difficult decision must be made by clinicians at the end of therapy, because clinical symptoms may be absent and laboratory tests may be normal in patients with active disease. The most confusing patients have residual disease, but in the meantime can present active disease or remission. Up to one-third of patients have continuous inflammatory lesions or subendothelial immune complexes despite complete clinical response. Meanwhile, over a half of patients with residual low-level proteinuria (500-1000 $\mathrm{mg} / 24 \mathrm{~h}$ ) after years of therapy do not appear to have active, ongoing renal inflammation. The rationale for renal biopsy before withdrawal of maintenance therapy is prevention of $L N$ relapses via identification of patients with ongoing, but clinically silent histological activity. The typical patient with $\mathrm{LN}$ - a young woman at reproductive age - requires from the physician special care with counselling and management from conception to labour. In all patients considering pregnancy in the period im- mediately after withdrawal of maintenance treatment of LN, the risk of subsequent LN flare is high. Thus, performance of renal biopsy in these patients is a reliable tool to establish LN activity. The presence of subendothelial immune deposits, cellular/fibrocellular crescents, glomerular necrosis, or active interstitial nephritis and a National Institute of Health (NIH) activity index > 2 [1], is the argument against stopping the treatment and in favour of changing the agent to a less toxic one for pregnancy (MMF to AZA).

On the other hand, repeat renal biopsy after the maintenance period allows one not only to distinguish patients with histopathological activity, but also to safely stop immunosuppression in patients with residual disease. All these considerations create the need for a prospective study randomizing patients to continuation or withdrawal of maintenance immunosuppression on the basis of the renal biopsy result.

Almost all recommendations included repeat renal biopsy in a flare. Patients with non-proliferative LN class II or $\mathrm{V}$ benefit more from repeated renal biopsies, because they have a reasonable possibility to switch to a proliferative LN that may require more aggressive immunosuppression [2, 3]. In all cases of refractory or bad response to the treatment connected with LN progression, repeat renal biopsy is strongly recommended.

In conclusion, all the evidence from studies and personal experience should be compared with our daily practice, where not only the patient, but also doctors are afraid of invasive procedures. Despite the low incidence of side effects caused by renal biopsies, the fear of unnecessary pain or complications still leads to delay in

Address for correspondence:

Ewa Haładyj, Department of Connective Tissue Diseases, National Institute of Geriatrics, Rheumatology and Rehabilitation, Spartańska 1, 02-637 Warsaw, Poland, e-mail: ehaladyj@o2.pl

Submitted: 15.03.2016; Accepted: 10.04.2016 
performing the biopsy. The activity of LN cannot be completely estimated by the clinical picture or the laboratory tests - the only possibility is to assess the histopathological pattern of the kidney specimen. The awareness of this fact should be an important argument in favour of performing renal biopsy not only at diagnosis but also in a subsequent renal flare and after the maintenance treatment.

The authors declare no conflict of interest.

\section{References}

1. Austin HA 3rd, Muenz LR, Joyce KM, et al. Diffuse proliferative lupus nephritis: Identification of specific pathologic features affecting renal outcome. Kidney Int 1984; 25: 689-695.

2. Lee HS, Mujais SK, Kasinath BS, et al. Course of renal pathology in patients with systemic lupus erythematosus. Am J Med 1984; 77: 612-620.

3. Daleboudt GM, Bajema IM, Goemaere NN, et al. The clinical relevance of a repeat biopsy in lupus nephritis flares. Nephrol Dia Transplant 2009; 24: gfp359. 